\title{
Time Trade-Off Procedure for Measuring Health Utilities Loss With Human Papillomavirus-Induced Diseases: A Multicenter, Retrospective, Observational Pilot Study in Italy
}

Article in Clinical Therapeutics · August 2011

Impact Factor: 2.73 · DOI: 10.1016/j.clinthera.2011.06.012 · Source: PubMed

CITATIONS

5

14 authors, including:

Francesco Saverio Mennini

University of Rome Tor Vergata

93 PUBLICATIONS 281 CITATIONS

SEE PROFILE

\section{Marco Petrillo}

Catholic University of the Sacred Heart 74 PUBLICATIONS 657 CITATIONS

SEE PROFILE

\section{Donatella Panatto}

Università degli Studi di Genova

102 PUBLICATIONS 525 CITATIONS

SEE PROFILE

\section{Roberto Gasparini}

Università degli Studi di Genova 213 PUBLICATIONS 2,081 CITATIONS

SEE PROFILE 


\title{
Time Trade-Off Procedure for Measuring Health Utilities Loss With Human Papillomavirus-Induced Diseases: A Multicenter, Retrospective, Observational Pilot Study in Italy
}

\author{
Francesco Saverio Mennini, DE ${ }^{1}$; Donatella Panatto, $\mathrm{BS}^{2}$; Andrea Marcellusi, $\mathrm{MS}^{1}$; \\ Paolo Cristoforoni, $\mathrm{MD}^{3}$; Rosa De Vincenzo, $\mathrm{MD}^{4}$; Elisa Di Capua, $\mathrm{MD}^{3}$;

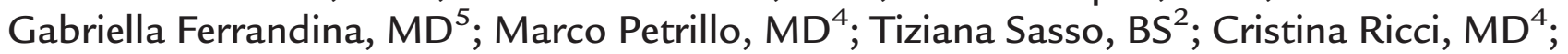 \\ Nausica Trivellizzi, $\mathrm{MD}^{4}$; Alessandro Capone, $\mathrm{MD}^{6}$; Giovanni Scambia, $\mathrm{MD}^{4}$; and \\ Roberto Gasparini, $\mathrm{MD}^{2}$
}

${ }^{1}$ Center for Health Economics and Management, Faculty of Economics, University Tor Vergata, Rome, Italy; ${ }^{2}$ Department of Health Sciences, University of Genoa, Genoa, Italy; ${ }^{3}$ Department of Oncologic Gynecology, National Institute for Cancer Research, Genoa, Italy; ${ }^{4}$ Department of Obstetrics and Gynecology, Catholic University of the Sacred Heart, Rome, Italy; ${ }^{5}$ Gynecologic Oncology Unit, Catholic University of the Sacred Heart, Campobasso, Italy; and ${ }^{6}$ Henley Centre for Value Improvement, Henley Business School, University of Reading, Reading, United Kingdom

\section{ABSTRACT}

Background: The economic evaluation of any human papillomavirus (HPV) vaccination strategy requires the measurement of clinical benefits (qualityadjusted life-years [QALY]) gained to reflect both the increase in life expectancy and the economic benefits associated with an effective intervention.

Objective: The purpose of this pilot study was to investigate the feasibility of a standardized time tradeoff (TTO) procedure to quantify utilities loss in health states affected by HPV-induced pathologies in Italy.

Methods: This multicenter, retrospective, observational, cross-sectional study was designed to elicit data on utilities in a cohort of women with a histologically confirmed diagnosis of high-grade cervical intraepithelial neoplasias (CIN2-3). An algorithm for the computerized administration of a TTO questionnaire was developed for the standardized elicitation of data on health utilities in CIN2-3, anogenital warts, and invasive cervical cancer. The European Quality of Life-5 Dimensions (EQ-5D) questionnaire was used to assess the respondents' baseline perception of their health conditions. The correlation between utilities and age, time from conization to questionnaire administration, and EQ-5D score, was tested using the Spearman rank correlation coefficient $(\rho)$ as a measure of validity.

Results: Of 42 enrolled patients, 36 responded $(85.7 \%)$ (mean [SD] age, 37.2 [9.0] years). The women's perception of their health state was high (mean [SD]
EQ-5D score, 0.93 [0.10]). The mean utility values were 0.73 (0.22), $0.71(0.35)$, and $0.02(0.08)$ for CIN2-3, anogenital warts, and invasive cervical cancer, respectively. Based on $\rho$ values, none of the $3 \mathrm{HPV}$-induced pathologies considered was significantly correlated with utility. Nonsignificant variability was found among utilities elicited for anogenital warts (range, 0.54 [0.47] to 0.79 [0.27]); this variability was a limitation of this pilot study and was likely the result of the limited sample size.

Conclusions: Based on the findings from this pilot study, a TTO standardized procedure is expected to be feasible and appropriate for assessing utilities in patients affected by HPV-related diseases and for cost-effectiveness analyses of cervical cancer prevention in Italy. (Clin Ther. 2011;33:1084-1095) (C) 2011 Elsevier HS Journals, Inc. All rights reserved.

Key words: anogenital warts, cervical cancer, CIN2-3, HPV, quality of life, TTO, utilities.

\section{INTRODUCTION}

Human papillomavirus (HPV) is a common cause of sexually transmitted infections that are generally transient, with up to $90 \%$ resolving within a couple of

Accepted for publication June 13, 2011.

doi:10.1016/j.clinthera.2011.06.012

0149-2918/\$ - see front matter

๑) 2011 Elsevier HS Journals, Inc. All rights reserved. 
years. ${ }^{1-3}$ When an infection is persistent, a variety of benign, premalignant, or malignant conditions, such as vaginal, vulvar, perianal, and cervical neoplasias, may occur in infected women, while anogenital warts may be diagnosed in both sexes. ${ }^{4,5}$ The initial significant consequence of persistent HPV infection is typically a low-grade cervical intraepithelial neoplasia (CIN1). Up to $33 \%$ of CIN1 cases progress to high-grade (CIN2-3) lesions, which are detected in $>10$ million women worldwide each year. ${ }^{6} \mathrm{CIN} 2-3$ lesions progress to invasive cervical cancer in $\sim 500,000$ women each year. Cervical cancer represents the leading cause of cancer-related mortality among women, with $\sim 230,000$ deaths per year worldwide. ${ }^{7,8}$

Depending on the outcome examined, the prevention of HPV-related disease with vaccination, together with cervical cancer screening programs, would provide substantial clinical, organizational, and economic benefits in short, intermediate, and long terms. The cost-effectiveness of HPV-virus-like particle vaccines has been reported in modeling studies ${ }^{9-21}$ that measured parameters such as life-years gained (LYG) or, more frequently, quality-adjusted LYGs (QALYs), for comparisons between health care interventions or predetermined thresholds. The combination of an efficacy indicator (LYG) with a quality indicator (health-related quality of life) into a single measurement (QALY) may be helpful in driving decision-making processes. QALY is used by many regulatory agencies (eg, the National Institute for Health and Clinical Excellence) as an integral part of health technology assessment methods and procedures. ${ }^{22}$ However, the QALY is closely related to the individual (or population) utility that can be attributed to the recovery or preservation of a healthy state.

The QALY is mainly used to adjust for life expectancy gained on the basis of the health-related quality of life predicted throughout the lifetime or part of it. Pliskin et $\mathrm{al}^{23}$ were the first to develop a mathematical model for the concept of QALYs that included all recognized key elements of general medical outcomes. ${ }^{23}$ The QALY-maximization criterion was founded on a multiattribute utility theory that requires the following conditions: utility independence between life-years and health status; risk neutrality on life-years; and constant proportional tradeoff behavior. ${ }^{23}$ In this framework, many social and cultural factors (eg, age, sex, underlying comorbidities, economic status, lifestyle, education, cognitive capabilities, organizational models, religion) may affect the health-state preferences of patients. ${ }^{24-26}$ Although collected with an identical methodology, different values of QALY have been obtained in several countries. ${ }^{27}$ In a comparison involving 7 studies conducted to assess the mean utility for end-stage renal disease, Arnesen and Trommald ${ }^{27}$ found that results varied up to 2.15-fold compared with the lower bound.

Based on the model of Pliskin et al, ${ }^{23}$ Miyamoto and Eraker $^{28}$ reshaped the formal equation to calculate QALYs, as follows:

$$
\text { QALYs }=b \cdot \mathrm{L}^{\mathrm{r}} \cdot \mathrm{U}
$$

with $U \leq 1$, where $L$ are the expected life-years, $b$ is a scaling constant (proportional trade-off), and $r$ is a risk parameter (risk neutrality). Although the risk parameter can assume any value, it usually is $0.5<r<1.0$, indicating risk aversion. Sometimes $r$ may be known, but in most circumstances this parameter must be estimated. However, because the computation of the riskaversion parameter $(r)$ is not easy, the risk-adjusted QALY is occasionally used in current applications. A practical approach is to assume that the utility function over life-years is linear $(r=1)$. $U$ is a measure of utility (corresponding to an individual's preference for a specific health state compared with alternative health states), with numerical values ranging between 0 (death) and 1 (optimal or "perfect" health). Some health states may be considered worse than death and thus may be assigned negative values. Simplifying the earlier equation, the number of QALYs lived by an individual in 1 year is calculated as:

$$
\text { QALYs }=1 \cdot \mathrm{U}
$$

However, QALYs as a criterion for evaluating medical interventions are calculated in the framework of costeffectiveness analyses. In this context, the value of QALYs gained is:

$$
\begin{aligned}
\text { QALYs }=\left(\mathrm{a}+\mathrm{L}^{\mathrm{i}} \Sigma \mathrm{t}\right. & =\mathrm{a})\left(\mathrm{U}_{\mathrm{t}}^{\mathrm{i}} /[1+\mathrm{r}]^{\mathrm{t}-\mathrm{a}}\right) \\
& -(\mathrm{a}+\mathrm{L} \Sigma \mathrm{t}=\mathrm{a})\left(\mathrm{U}_{\mathrm{t}} /[1+\mathrm{r}]^{\mathrm{t}-\mathrm{a}}\right),
\end{aligned}
$$

where $L$ denotes the duration of the disease, $L^{i}$ is the treatment period, and $U^{i}$ is the vector of utilities predicted (better if measured) for each time period $t$ after the intervention undertaken at age $a .^{29}$ This equation includes the concept of quality-adjusted life expectancy (QALE) because QALYs are used to measure the improvement in QALE gained through a specific health intervention compared with a standard alternative intervention. 
To provide the most appropriate health care services, policymakers should base their decisions on reliable economic evaluations. Utilities assessments and the collection of QALY values specifically to each country (or to geographic areas with similar socioeconomic characteristics) are urgently needed.

Because utilities have been measured and are consistently used (ie, QALYs and related threshold values) in Europe (in the United Kingdom and in a district of Spain exclusively), considering that patients' health preferences are relevant to decision-making processes that aim to allocate resources, and because the costeffectiveness of HPV vaccination currently deserves further appropriate evaluation, the objective of the present pilot study was to assess the feasibility of a time trade-off (TTO) standardized procedure to quantify utilities loss in health states affected by HPV-related pathologies in Italy.

\section{METHODS}

\section{Study Design}

This multicenter, observational, retrospective, crosssectional pilot study was part of a larger research project comprising 4 studies. It was designed to elicit healthstate preferences (utilities) in a cohort of patients with a histologically confirmed diagnosis of CIN2-3. Among HPV-related diseases (eg, invasive cervical cancer), it was expected that such lesions would have been perceived with a wide variability in affecting the patients' quality of life.

Because spontaneous regression is rare in CIN2-3, patients were referred to a colposcopy clinic, where the lesions were examined using optical equipment, biopsy samples were obtained, and the involved portion of the cervix was removed using conization. Conization was carried out in hospital setting (in-hospital or day-hospital), where informed consent is mandatory. Although sometimes asymptomatic, patients generally experience psychological stress associated with the risk for progression to invasive cancer. In addition, patients may be concerned about the potential transmission of infection to their partners and future pregnancies.

Three clinical research centers, each with its own archive of patient records and located in northern, central, or southern Italy, participated in this study: the Department of Oncologic Gynecology, National Institute for Cancer Research, Genoa; the Department of Obstetrics and Gynecology, Catholic University of the Sacred Heart, Rome; and the Gynecologic Oncology
Unit, Catholic University of the Sacred Heart, Campobasso. The study was approved by the local institutional review board in Genoa, and notification was given to the related ethics committee. On the basis of current official guidelines enacted by the National Regulatory Agency ${ }^{30}$ for noninterventional observational studies conducted in Italy, notification provided to the ethics committee is satisfactory. A further in-depth evaluation of the study protocol was conducted by the Italian Ministry of Education, University and Scientific Research, which provided economic support for the implementation of the pilot study.

All personal data (name, date of birth, and personal health code) were replaced by a univocal numerical code to make all clinical records unidentifiable (in full compliance with the privacy law in Italy). The retrospective nature of the study design did not require the collection of any further informed consent.

In addition to the assessment of utilities loss with HPV-related diseases, this pilot study was designed to investigate 3 secondary objectives: (1) on the basis of utilities distribution, to appraise the validity of the procedure, taking into account the effects of the following variables: age, quality of life perceived at baseline (measured using the European Quality of Life-5 Dimensions [EQ-5D] questionnaire [EuroQol Group, Rotterdam, the Netherlands]), time from conization to administration of questionnaires, and mean time taken for completion of the questionnaire among research centers; and (2) to calculate the mean and the variance of utilities associated with the diagnosis of CIN2-3, which are required to estimate the optimal sample size to conduct a broader and statistically representative study concerning the Italian population; and (3) and to assess the feasibility of this method for measuring utilities lost with other HPV-induced diseases that did not directly affect enrolled patients (eg, anogenital warts, invasive cervical cancer).

\section{Patient Population}

Patients eligible for enrollment met the following criteria: age between 18 and 65 years at the time of diagnosis; a histologically confirmed diagnosis of CIN2-3; a recorded surgical procedure of hospital conization; and time from conization to administration of questionnaires $<78$ weeks (to avoid the potential impairment effect of recollection that might be associated with a stressor event). All consecutive patients recorded in the computerized archives at the clinical 


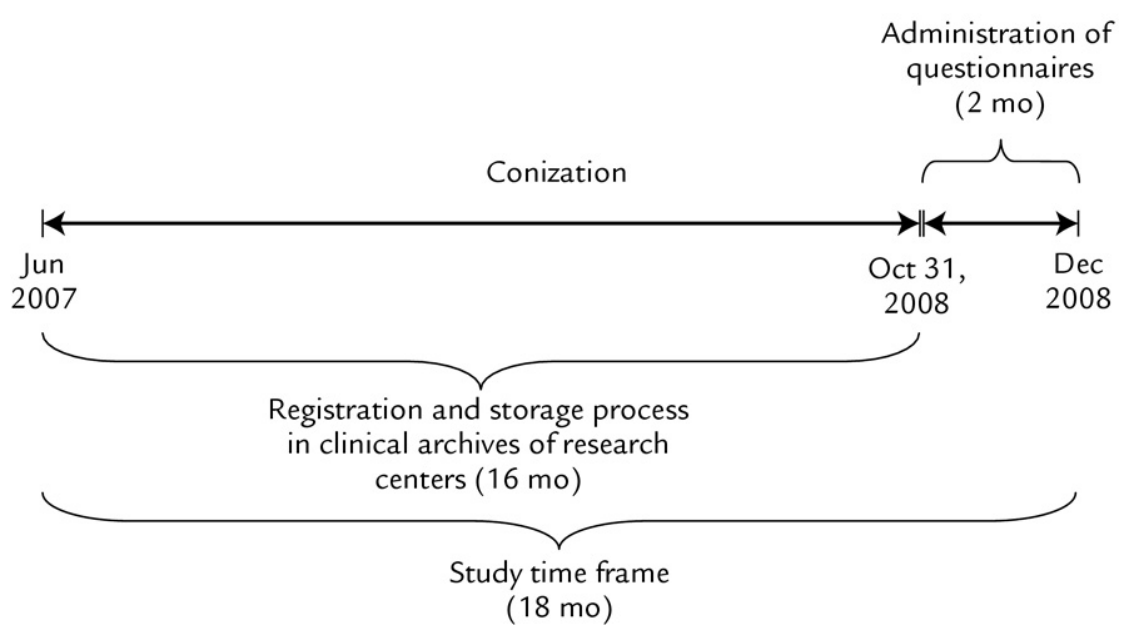

Figure 1. Design and observation time frame in this study of the use of a time trade-off standardized procedure for eliciting health utilities in women with human papillomavirus-related diseases.

centers within the 16 months preceding the end of October 2008 could be potentially eligible as respondents. The enrollment procedure was suspended in December 2008, when the sample size matched a level corresponding to $\sim 10 \%$ of overall CIN2-3 patients listed in the clinical archives. This sample size was calculated to provide a rational confidence level in assessing the feasibility of the standardized TTO procedure exclusively. The percentage was also based on the analysis of 2 previous publications that evaluated data recorded in reliable and representative Italian databases on cervical cancer screening and cervical cancer registry. ${ }^{31,32}$

The study design and associated time frame are presented in Figure 1. Patients who did not fully complete both questionnaires used in this pilot study (measuring utilities and quality of life) and those with an incomplete clinical record were excluded.

\section{Utility Elicitation Procedure}

The TTO procedure was developed by Torrance et $\mathrm{al}^{33-35}$ based on a less complicated but conceptually equivalent method comparable to standard gamble (SG). Like SG, TTO was based on trade-offs, in which respondents "trade off" survival time and health states. The utility value is measured by finding the point at which the respondent cannot choose between 2 scenarios (indifference). In cases of intermediate-term and chronic diseases, the choice is generally between the illness for a period of time and perfect health for a shorter time, both followed by death. In contrast, in short-term illnesses, the choice is between the illness for a period of time and a worse health state for a shorter time, both followed by the same specified outcome.

In the conventional implementation, the first alternative offers a suboptimal steady health state $(i)$ with a given duration $(t)$. A better health state (commonly perfect health) of shorter duration $(x)$ is offered as the competing alternative. Both health states are followed by death. The point of indifference $\left(b_{i}\right)$ is reached by varying the duration spent in perfect health. Subsequently, by combining $x$ and $t$, the value of the steady health state is established as follows:

$$
\mathrm{h}_{\mathrm{i}}=\mathrm{x} / \mathrm{t} \text {, }
$$

where $x<t$.

In cases of short-term diseases, the formal equation to achieve the point of indifference is calculated as:

$$
\mathrm{h}_{\mathrm{i}}=1-\left(1-\mathrm{h}_{\mathrm{j}}\right) \cdot \mathrm{x} / \mathrm{t}
$$

On the basis of TTO procedure, utilities are cardinal figures representing the strength of patients' preferences for particular outcomes. According to a reference-based guideline to design and produce health-state utility instrumentations, ${ }^{36}$ an algorithm committed to specifically to the computer-guided administration of a questionnaire was developed for the standardized elicitation of healthstate preferences in patients with HPV-related CIN2-3 lesions (see Supplemental Figure 2 in the online version at doi:10.1016/j.clinther.2011.06.012). The computerized 
method to elicit utilities was also realized to ensure an entry and automatic transmission of data to dedicated and protected electronic archives that were stored in predetermined computers of each research center.

The women enrolled underwent a computerguided face-to-face interview in which a series of 2 alternatives were presented, and the patients were asked to choose the one that they preferred. Alternative A was a pathologic condition (eg, CIN2-3, invasive cervical cancer, anogenital warts) that might have been experienced for the remaining life expectancy (on the basis of data by age and sex provided by the Italian National Institute of Statistics ${ }^{37}$ ). Alternative B was a shorter period of time which would have been lived in a full healthy state, followed by death. The duration of time spent in alternative $\mathrm{B}$ was varied (depending on the disease) until the achievement of the point of indifference.

For the elicitation of individual responses, conditions were presented using a written description and photos. Patients were also shown a bar (proportionally divided into "good" and "bad" health segments) to indicate clearly the number of years to trade-off, depending on the patient's age (see Supplemental Figure 3 in the online version at doi:10.1016/j.clinther.2011.06.012). The EQ-5D was used to assess patients' perception of health status at baseline, using 5 dimensions: mobility, self-care, usual activities, pain/discomfort, and anxiety/depression. ${ }^{38}$ Within each dimension were 3 levels of severity: no problems; moderate problems; and severe problems (see Supplemental Figure 4 in the online version at doi:10.1016/j.clinther.2011.06.012).

The computer-guided administration of questionnaires was preceded by an oral explanation about the nature and objectives of the study to avoid any misconception in patients and possible mistakes. Interviewers were provided with a written guideline and a time interval (9-15 minutes) required to accomplish the elicitation of utilities to control and ensure that the implementation of TTO was a totally fulfilled standardized procedure. To neutralize the confounding effect of a deviating administration of questionnaires among research centers, interviewers underwent 2 normalizing training sessions.

Although health-state preferences should be sequentially evaluated starting from the worst condition to the best, ${ }^{25}$ utilities were also calculated for pathologies such as invasive cervical cancer (an indisputably worse condition compared with CIN2-3) and anogenital warts, which did not directly affect respondents.

\section{Statistical Analysis}

All continuous data are expressed means (SD), and 95\% CI is provided when appropriated. Considering a hypothetically small sample size, nonparametric tests were performed to avoid any predetermined assumption on variables distribution (age, time from conization to administration of questionnaires, and time taken for the completion of questionnaires). Differences between utilities in study centers were tested with the Kruskal-Wallis 1-way ANOVA by rank. The correlation between utilities and age, EQ-5D score, and time from conization to administration of questionnaires was tested using the Spearman's rank correlation coefficient $(\rho)$. Both tests and $\rho$ in particular were performed as measuring the criterion validity of the adopted procedure. Statistical significance was set at $\alpha<5 \%$, and all analyses were conducted using SPSS version 17 (SYSTAT, Chicago, Illinois).

\section{RESULTS}

Of 42 patients screened, 36 participated, providing an overall response rate of $85.7 \%$. All analyses were performed on the sample of patients who completed the TTO and EQ-5D ( $\mathrm{n}=36$; mean [SD] age, 37.2 [9.0] years). The characteristics of the respondents are summarized in Table I. Patients interviewed in Campbasso had a significantly shorter mean time from conization to administration of questionnaires (32.8 [21.0] weeks; $P=0.030$ ). At the time of the interview, the mean (SD) EQ-5D score (perception of their health state) was 0.93 (0.10) (high).

None of the 36 respondents refused to trade off a lifetime for perfect health states given. The mean utility values by $\mathrm{HPV}$-induced pathologies were $0.73(0.22)$, 0.71 (0.35), and 0.02 (0.08) for CIN2-3, anogenital warts, and invasive cervical cancer, respectively (Figure 2). The mean utility values for the 3 research centers by $\mathrm{HPV}$-induced disease are reported in Table II. With the computer-guided administration of the TTO questionnaire, the mean utility values did not differ significantly between research centers on Kruskal-Wallis 1-way ANOVA. Variability was found in the utility values for anogenital warts between research centers (range, 0.54 [0.47] to 0.79 [0.27]), but this variability did not reach statistical significance. 
Table I. Characteristics of the respondents in this study of the use of a time trade-off standardized procedure for eliciting health utilities in women with human papillomavirus-related diseases.

\begin{tabular}{|c|c|c|c|c|c|}
\hline Characteristic & $\begin{array}{c}\text { Genoa } \\
(n=20)\end{array}$ & $\begin{array}{c}\text { Rome } \\
(n=11)\end{array}$ & $\begin{array}{c}\text { Campobasso } \\
(\mathrm{n}=5)\end{array}$ & $\begin{array}{l}\text { All Patients } \\
(\mathrm{N}=36)\end{array}$ & $P^{*}$ \\
\hline \multicolumn{6}{|l|}{ Age, $y$} \\
\hline Mean (SD) & $38.5(8.4)$ & $35.1(9.6)$ & $36.8(11.4)$ & $37.2(9.0)$ & 0.486 \\
\hline Range & $24-52$ & $21-60$ & $24-53$ & $21-60$ & \\
\hline $\begin{array}{l}\text { Time elapsed, }^{\dagger} \\
\text { mean (SD), wk }\end{array}$ & $36.1(20.9)$ & $35.3(20.7)$ & $13.8(10.4)$ & $32.8(21.0)$ & 0.030 \\
\hline Time taken, ${ }^{\ddagger}$ mean $(S D)$, min & $8.3(2.3)$ & $10.6(1.8)$ & $8.6(2.4)$ & $8.8(2.3)$ & 0.061 \\
\hline EQ-5D score, mean (SD) & $0.96(0.1)$ & $0.90(0.2)$ & $0.91(0.1)$ & $0.93(0.1)$ & 0.749 \\
\hline
\end{tabular}

EQ-5D = European Quality of Life-5 Dimensions questionnaire.

* Kruskal-Wallis 1-way ANOVA by rank.

†Time from conization to administration of questionnaires.

FTime for the completion of questionnaires.

In Table III, the effects of age, time from conization to administration of questionnaires, and EQ-5D score at baseline are considered with respect to the standardized computer-guided elicitation of patients' healthstate preferences. On analysis of $\rho$ values, none of the 3 considered variables were correlated with the utility measurements between research centers. Although far

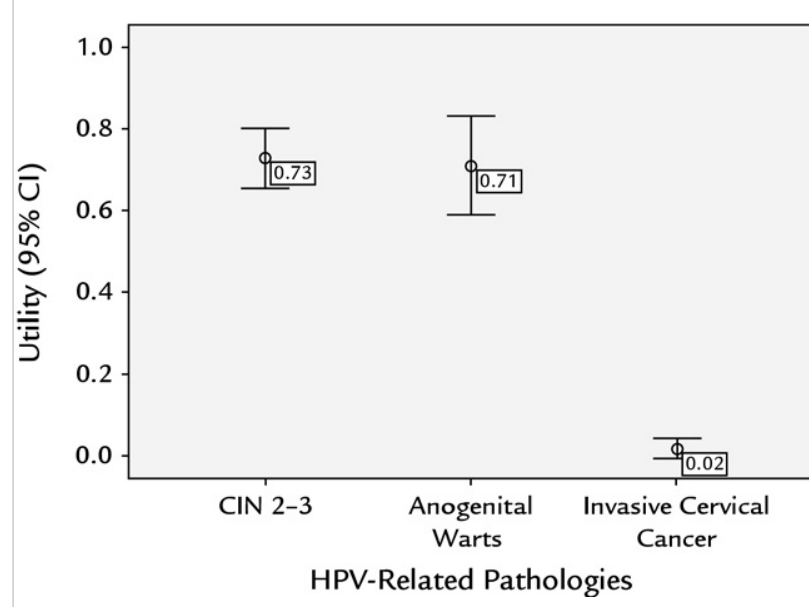

Figure 2. Utilities elicited with the computerguided administration of the time tradeoff procedure in this study in women with human papillomavirus (HPV)-related diseases $(\mathrm{N}=36)$. from the achievement of statistical significance, the variable time elapsed before the questionnaire administration in patients with CIN2-3 may have slightly affected the reported utility values. The variability found with anogenital warts was large but not statistically significant (95\% CI, 0.22-0.85; $P=0.493$ ).

Based on the variability of utility distribution values collected for a pathologic condition such as CIN2-3 (Figure 3), the sample size required to have an error value of $\theta=0.05$ and a significance level of $\alpha=0.05$ was calculated. Given the parameters of the investigation (SD = 0.2 ), the minimum sample size required to estimate utilities in a larger and statistically representative Italian female population was 360 patients; 60 for each evaluated pathologic condition (atypical squamous cells of undetermined significance, CIN1, CIN2-3, invasive cervical cancer, and anogenital warts), together with a control group of 60 healthy age-matched patients.

To estimate resources needed, to minimize the organizational issues associated with the design of a study involving a larger population, and to control that the future implementation of computer-guided TTO would be a totally standardized procedure, the accomplishment of utilities elicitation took a mean (SD) time of 8.8 (2.3) minutes. Within the 3 research centers there was no significant difference related to time taken for health-related utility assessment (8.3 [2.3], 10.6 [1.8], and 8.6 [2.4] minutes in Genoa, Rome, and Cam- 
Table II. Utility measurement in this study of the use of a time trade-off standardized procedure for eliciting health utilities in women with human papillomavirus (HPV)-related diseases.

\begin{tabular}{lcccc} 
HPV-Related Disease & $\begin{array}{c}\text { Genoa } \\
(\mathrm{n}=20)\end{array}$ & $\begin{array}{c}\text { Rome } \\
(\mathrm{n}=11)\end{array}$ & $\begin{array}{c}\text { Campobasso } \\
(\mathrm{n}=5)\end{array}$ & $P^{*}$ \\
\hline CIN2-3 & & & & 0.809 \\
Mean (SD) & $0.72(0.23)$ & $0.74(0.24)$ & $0.73(0.11)$ & 0.75 \\
Median & 0.80 & 0.85 & $0.59-0.87$ & 0.493 \\
95\% Cl & $0.61-0.83$ & $0.58-0.90$ & & $0.77(0.32)$ \\
Anogenital warts & & & 0.91 & 0.097 \\
Mean (SD) & $0.79(0.27)$ & $0.54(0.47)$ & $0.37-1.00$ & \\
Median & 0.91 & 0.50 & $0.00(0.00)$ & 0.00 \\
95\% Cl & $0.66-0.91$ & $0.22-0.85$ & $0.00-0.00$ & \\
Cervical cancer & & &
\end{tabular}

CIN2-3 = high-grade cervical intraepithelial neoplasias.

*Kruskal-Wallis 1-way ANOVA by rank.

pobasso, respectively). Measurements of utilities related to other HPV-induced diseases (anogenital warts, invasive cervical cancer) was carried out without any additional time. It is logical that the utilities elicited in this pilot trial cannot be immediately implemented in studies focused on cost-utility analysis or cost-effectiveness analysis. This requires that utilities are collected in a larger number of patients with HPV-induced diseases through the computer-guided administration of the TTO standardized procedure.

\section{DISCUSSION}

In these economically turbulent times, health policy decisions are made according to appropriate procedures and considering the comparative evaluation of new and existing technologies for given diseases such as HPV-induced diseases (eg, anogenital warts, precancerous lesions, and invasive cervical cancer). The evaluation of health programs is a part of health technology assessment research. Whether efficiency is the

Table III. Validity of the time trade-off procedure, measured by correlation coefficient, for eliciting health utilities in women with human papillomavirus (HPV)-related diseases $(\mathrm{N}=36)$.

\begin{tabular}{|c|c|c|c|c|c|c|}
\hline \multirow[b]{2}{*}{ HPV-Related Disease } & \multicolumn{2}{|c|}{ Age } & \multicolumn{2}{|c|}{ Time Elapsed* } & \multicolumn{2}{|c|}{ EQ-5D } \\
\hline & $\rho$ & $P$ & $\rho$ & $P$ & $\rho$ & $P$ \\
\hline CIN2-3 & -0.079 & 0.645 & -0.283 & 0.095 & -0.063 & 0.716 \\
\hline Anogenital warts & -0.070 & 0.686 & -0.222 & 0.192 & 0.039 & 0.823 \\
\hline Cervical cancer & 0.223 & 0.191 & -0.002 & 0.992 & -0.107 & 0.533 \\
\hline
\end{tabular}

$\rho=$ Spearman's rank correlation coefficient; CIN2-3 = high-grade cervical intraepithelial neoplasias; EQ-5D = European Quality of Life-5 Dimensions questionnaire.

*Time elapsed between conization and questionnaire administration. 


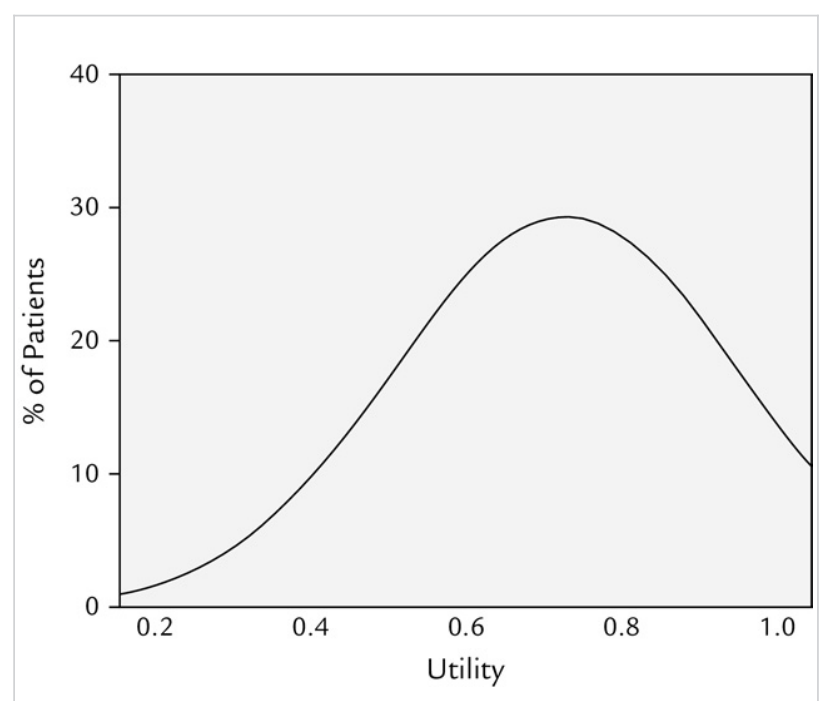

Figure 3. Distribution of utility values for high-grade cenvical intraepithelial neoplasias in this study of the use of a time-trade-off standardized procedure for eliciting health utilities in women with human papillomavirus-related diseases $(\mathrm{N}=36)$.

guiding principle of decision-making process, cost-effectiveness analysis and cost-utility analysis are the preferred strategies. ${ }^{39,40}$ Both strategies incorporate the costs of treatment and express the added value of health interventions with additional resources required to increase the outcome measured at baseline (eg, LYGs). As a result, regulatory agencies approve new expensive health care treatments, devices, or primary prevention programs (eg, vaccination) and the licensing of new drugs on the basis of health technology assessments, including cost-utility analysis, with national and international perspectives. ${ }^{22,41}$ Cost-utility and cost-effectiveness analyses take into account utility values in each assessment; however, the validity of a cost-utility analysis depends on the validity of its components. Because there is no a "gold standard" for utilities, different elicitation methods may produce different utilities from the same respondents. More important, utilities elicited for the same disease do not have exactly the same values in all countries.

Arnesen and Trommald ${ }^{26}$ reviewed the findings from several TTO studies conducted with the same methodology for eliciting utilities from the patient's perspective. As depicted in Figure 4, utilities for endstage renal disease in patients undergoing hemodialysis in a hospital setting ranged from 0.39 to 0.84 in the selected publications. This large variability may have been associated with many parameters of study design and methodology. The SDs were generally large, and

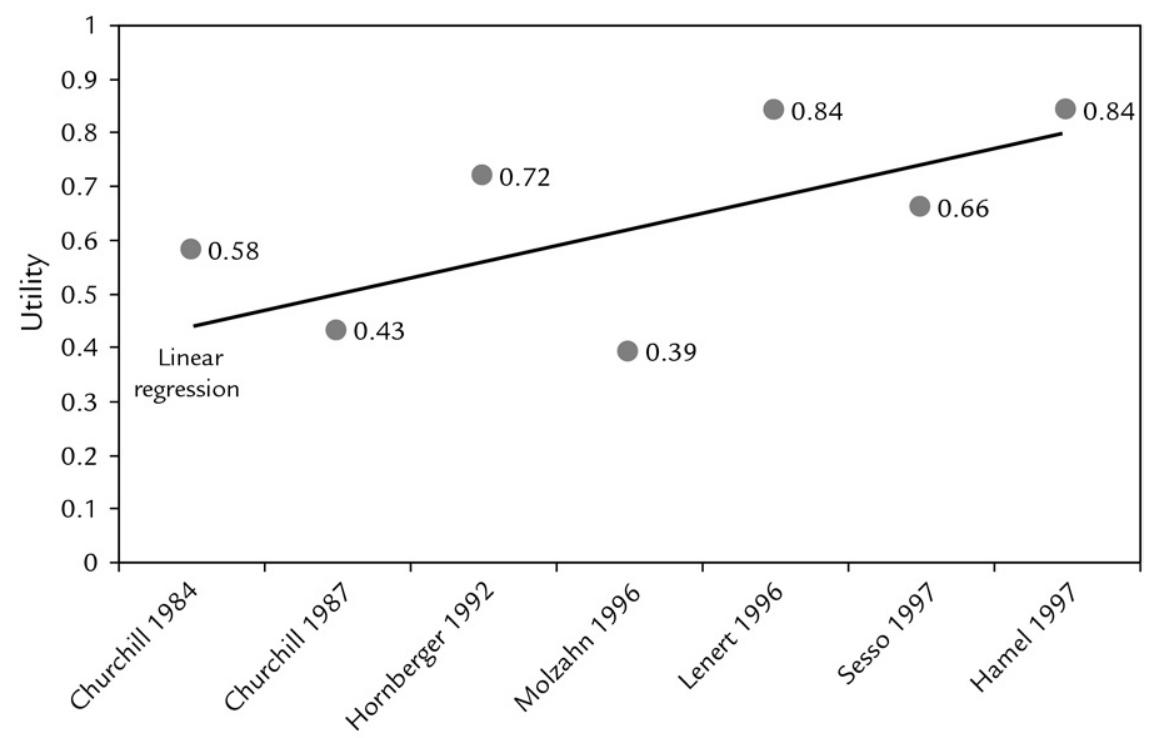

Figure 4. Utility values in a previously published study in hospitalized patients with end-stage renal disease. $^{28,42-48}$ 
most of the research groups used complete samples, but the response rates varied greatly. The TTO was administered as questionnaires or as personal, structured, semistructured, or computer-assisted interviews. The influence of quality of life at baseline, age, and sex on the utility values was not systematically considered. The hypothetical life expectancy used as a time horizon for the trade varied significantly between the studies. Doubtless, the wide variation in results found for hemodialysis is representative for the methodological approach. The comparison of elicited utility values was even more complicated when populations from different countries were considered. The utility assessment can be influenced by several factors basically related to social and cultural issues, which should be added to specific potential procedural bias, including health-state descriptions, cognitive burden, and interviewer bias.

The health-state description might not be an issue if a respondent previously experienced health problems induced by a particular disease. In contrast, a hypothetical health state requires narrations and descriptions that can be positively or negatively framed, and these descriptions may vary significantly between interviewers. ${ }^{49,50}$ Orally provided descriptions may overload or weaken the memory of respondents. Such confounding effects may be avoided by providing a written health-state description that is detailed either from a positive or a negative perspective, and graphic illustrations together with text to provide standard and neutral information and to facilitate memory recall.

The elicitation of utilities can also be a cognitive challenge. Respondents may have difficulty with numeracy or with the comprehension of proportional remaining life-years to trade-off. ${ }^{51}$ Such inconveniences may be addressed with a careful and standardized explanation, with simple and self-explanatory figures, and by testing the comprehension of respondents with regard to the questionnaires. Computerized elicitation methods may improve the assessment of utilities. ${ }^{52,53}$ Interviewers have reported user-friendly administration and patients' full comprehension of the entire TTO procedure, with none of the patients reporting any misunderstanding or complaint.

A TTO method with a human assessor who explains each step and addresses questions stimulating answers is subject to interviewer bias. As previously mentioned, this bias may be surmounted with careful training of interviewers and systematic control in the adherence to the protocol. Presumably, the best approach is represented by automated elicitation, which may prevent many of the concerns of interviewer bias.

A literature search did not identify any previously published studies that analyzed utilities loss using a computer-guided administration of a TTO procedure for the standardized elicitation of health-state preferences in Italy. The findings from the present multicenter pilot study suggest feasibility and appropriateness of a TTO standardized procedure to assess utilities in patients affected by CIN2-3, anogenital warts, and/or invasive cervical cancer. The computer-guided administration of TTO questionnaires together with visual aids and specific education of interviewers may have ensured a homogeneous pattern of administration, may have been comprehended by patients, and may have led to a lack of data-entry errors and calculation errors. The findings from the present pilot study allowed for an estimation of resources (time and personnel) required for optimal planning and implementation of a larger study, which is expected to involve 360 women. Regardless of the specific pathology considered, the EQ-5D was found to be effective in assessing the general health-state preferences of the population studied.

There were some caveats about this pilot study. Although the TTO procedure is widely used for measuring patients' health-state preferences, it is not always possible to determine construct validity because utilities may legitimately change over time. They can be affected by different sociocultural and economic factors and by health care settings. Validity and reliability are 2 basic requirements for such evaluations. Although considered as 2 distinct methodological concepts, the boundary between validity and reliability is mathematically and conceptually blurred. The term validity suggests a method that can effectively measure what it was designed to measure. For subjective issues, such as health-state preferences, validity testing is not easy, especially considering the lack of a gold standard. The achievement of reliability is to some extent a technical matter; in contrast, validity is much more than the implementation of the appropriate technicality. Because it is related to the nature of the unit measured, validity is closely connected with the essence of procedure itself. ${ }^{54}$ In the present study, based on the analysis of correlation coefficients between research centers, an assessment of the criterion validity of the TTO procedure was performed. Although age, EQ-5D score, and 
time from conization to administration of questionnaires were not found to be correlated with the elicited utility values, variability was found with anogenital warts (range, 0.54-0.79). Despite that the number of enrolled patients with CIN2-3 lesions matched the predefined $10 \%$ rate of those listed in the clinical archives, the sample size might not have been completely adequate to detect statistically significant differences concerning anogenital warts. Utilities elicited for invasive cervical cancer might be deemed similar to the lowest limit of expected values. However, health is the most important aspect of life to some patients, who would prefer to live a short life in perfect health rather than trade for a long life with severe health problems. ${ }^{55}$ This idea supports that utilities should be calculated on the basis of time preferences elicited from patients who have actually experienced the pathologic condition being analyzed.

\section{CONCLUSION}

Based on the findings from this pilot study, a TTO standardized procedure is expected to be feasible and appropriate for assessing utilities in patients affected by HPV-induced diseases and for cost-effectiveness analyses of cervical cancer prevention in Italy.

\section{ACKNOWLEDGMENTS}

This research was financially supported by the Italian Ministry of Education, University and Scientific Research in Italy.

The authors acknowledge the support and relevant contributions of Prof. Mike Drummond and Prof. Bengt Jonsson, who were involved in the extensive and rapid peer review of this article.

The authors have indicated that they have no other conflicts of interest with regard to the content of the article.

Mr. Mennini has received research funding from Roche, Novartis, Merck Serono, Sanofi Psteur MSD, Abbott, Bristol-Myers Squibb, Pfizer, AstraZeneca, GlaxoSmithKlein. Mr. Marcellusi has received consulting fees from Merck Serono. Dr. Capone has received consulting fees from Bristol-Myers Squibb, Pfizer, Merck Sharp \& Dohme, AstraZeneca, sanofi-aventis, Serono, GlaxoSmithKlein, Lundbeck, Boehringheringellheim, Sanofi Pasteur MSD.

Mr. Mennini was the guarantor of the paper, conception and design, analysis and interpretation of data, drafting the article, final approval of the version to be published. Mr. Marcellusi, Dr. Capone, and Ms. Panatto were responsible for the conception and design, analysis and interpretation of data, drafting the article. Drs. Scambia and Gasparini were responsible for the analysis and interpretation of data, revising it critically for important intellectual content, final approval of the version to be published. Drs. Cristoforoni, De Vincenzo, Di Capua, Ferrandina, Petrillo, Ricci, Trivellizzi, and Ms. Sasso all participated in the data collection.

\section{SUPPLEMENTARY MATERIAL}

Supplementary material accompanying this article can be found in the online version at doi:10.1016/j.clinther. 2011.06.012.

\section{REFERENCES}

1. Bosch FX, Lorincz A, Munoz N, et al. The causal relation between human papillomavirus and cervical cancer. J Clin Pathol. 2002;55:244-265.

2. Snijders PJ, Steenberger RD, Heideman DA, Meijer CJ. HPV-mediated cervical carcinogenesis: concepts and clinical implications. J Pathol. 2006;208:152-164.

3. Weinstock H, Berman S, Caces W Jr. Sexually transmitted diseases among American youth: incidence and prevalence estimates, 2000. Perspect Sex Reprod Health. 2004;36:6-10.

4. Munoz N, Bosch FX, de Sanjose S, et al. Epidemiologic classification of human papillomavirus types associated with cervical cancer. NEnglJ Med. 2003;348:518-527.

5. de Villiers EM, Fauquet C, BrokerTR, et al. Classification of papillomaviruses. Virology. 2004;324:17-27.

6. Lowy DR, Frazer IH. Chapter 16: Prophylactic human papillomavirus vaccines.J Nat/ Cancer Inst Monogr. 2003;31:111-116.

7. Ferlay J, Bray F, Pisani P, Parkin DM, for the Descriptive Epidemiology Group of International Agency for Research on Cancer. Globocan 2002: Cancer incidence, mortality and prevalence worldwide. Release September 16, 2005. http://www-dep.iarc.fr. Accessed September 2009.

8. Cutts FT, Franceschi S, Goldie S, et al. Human papillomavirus and HPV vaccines: a review. Bull World Health Organ. 2007;85:719-726.

9. Sanders GD, Taira AV. Cost effectiveness of a potential vaccine for human papillomavirus. Emerg Infect Dis. 2003;9:37-48.

10. Kulasingam SL, Myers ER. Potential health and economic impact of adding a human papillomavirus vaccine to screening programmes. JAMA. 2003;290:781-789.

11. Taira AV, Neukermans CP, Sanders GD. Evaluating human papillomavirus vaccination programmes. Emerg Infect Dis. 2004;10:1915-1923.

12. Goldie SJ, Kholi M, Grima D, et al. Projected clinical benefits and cost-effectiveness of a human papillomavirus 16/18 vaccine. J Nat/ Cancer Inst. 2004;96:604-615. 
13. Barnabas RV, Laukkanen $P$, Koskela $\mathrm{P}$, et al. Epidemiology of HPV 16 and cervical cancer in Finland and the potential impact of vaccination: mathematical modelling analyses. PLoSMed. 2006;3:624-632.

14. Elbasha EH, Dasbach EJ, Insinga RP. Model for assessing human papillomavirus vaccination strategies. Emerg Infect Dis. 2007;13:28-41.

15. Favato G, Pieri V, Mills R. Costeffective analysis of anti-HPV vaccination programme in Italy: a multicohort Markov model. http:// www.ssrn.com/abstract $=961847$. Accessed October 2009.

16. Chesson HW, Ekwueme DU, Saraiya M, Markowitz LE. Cost-effectiveness of human papillomavirus vaccination in the United States. Emerg Infect Dis. 2008;14:244-251.

17. Jit M, Choi YH, Edmunds WJ. Economic evaluation of human papillomavirus vaccination in the United Kingdom. BMJ. 2008;337:a769.

18. Kim JJ, Goldie SJ. Health and economic implications of HPV vaccination in the United States. N Engl JMed. 2008;359:821-832.

19. Kim JJ, Ortendahl J, Goldie SJ. Costeffectiveness of human papillomavirus vaccination and cervical cancer screening in women older than 30 years in the United States. Ann Intern Med. 2009;151:538-545.

20. Mennini FS, Giorgi Rossi P, Palazzo F, Largeron N. Health and economic impact associated with a quadrivalent HPV vaccine in Italy. Gynecol Oncol. 2009;112:370-376.

21. Mennini FS, Costa S, Favato G, Picardo M. Anti-HPV vaccination: A review of recent economic data for Italy. Vaccine. 2009;27:A54-A61.

22. National Institute for Health and Clinical Excellence. Guide to the methods of technology appraisal (reference N0515). http://www. nice.org.uk/niceMedia/pdf/TAP_ $>$ Methods.pdf. Accessed September 2009.

23. Pliskin JS, Shepard DS, Weinstein MC. Utility functions for life years and health status. Operations Res. 1980;28:206-224.

24. Loomes G. Disparities between health state measures: is there a rational explanation? In: Bed G, ed. The Economics of Rationality. London, UK: Routledge; 1993:149-178.

25. Mehrez A, Gafni A. Preference based outcome measures for economic evaluation of drug interventions: quality adjusted life years (QALYs) versus healthyyears equivalents (HYEs). Pharmacoeconomics. 1992;1:338-345.

26. Sassi F, Archard L, Le Grand J. Equity and the economic evaluation of healthcare. Health Technology Assessment. 2001;5:1-138.

27. Arnesen T, Trommald M. Roughly right or precisely wrong? Systematic review of quality-of-life weights elicited with the time trade-off method. JHealth Serv Res Policy. 2004;9:43-50.

28. Miyamoto JM, Eraker SA. Parameter estimates for a QALY utility model. Med Decis Making. 1985;5:191-213.

29. Sassi F. Calculating QALYs, comparing QALY and DALY calculations. Health Policy Plan. 2006;21:402-408.

30. Italian Medicines Agency. Guidelines for classification and implementation of observational studies on drugs. http://www.agenziafarmaco.it/sites/ default/files/det_20marzo2008.pdf. Accessed September 2009.

31. Ferrandina G, Marcellusi A, Mennini FS, et al. Hospital costs incurred by the Italian National Health Service for invasive cervical cancer. Gynecol Oncol. 2010;119:243-249.

32. Costa S, Venturoli S, Mennini FS, et al. Population-based frequencyassessment of HPV-induced lesions in patients with borderline Pap tests in the Emilia-Romagna Region: the PATER study. Curr Med Res Opin. 2011;27:569-578.

33. Torrance GW, Tomas WH, Sackett DL. A Utility maximization model for evaluation of heath care programs. Health Serv Res. 1972;7:118-133.

34. Torrance GW. Social preferences for health states: an empirical evaluation of three measurement techniques. Socioeconomic Plan Sci. 1976;10:129-136.
35. Gafni A, Torrance GW. Risk attitude and time preference in health. Manage Sci. 1984;30:440 - 451.

36. Furlong W, Feeny D, Torrance GW, et al. Guide to design and development of health-state utility instrumentation. http://www.chepa.org/Files/ Working Papers/WP 90-9.pdf. Accessed September 2009.

37. The National Institute of Statistics. Italian Population Mortality Tables by province and region of residence. http://demo.istat.it/index_e.html. Accessed September 2009.

38. Rabin R, de Charro F. EQ-5D: a measure of health status from the EuroQol Group. Ann Med. 2001;33:337-343.

39. Gold MR, Siegel JE, Russel LB, Weinstein MC. Cost-Effectiveness in Health and Medicine. New York, NY: Oxford University Press; 1996.

40. Drummond MB, O'Brien B, Stoddart GL, Torrance GW. Methods for the Economic Evaluation of Health Care Programmes. Oxford, UK: Oxford University Press; 1997.

41. Australian Government, Department of Health and Ageing. Guidelines for preparing submissions to the Pharmaceutical Benefits Advisory Committee (Version 4.3), December 2008. http:// www.pbs.gov.au/pbs/pdf-viewer? $\mathrm{pdf}=\% 2$ Findustry\%2Flisting\%2Fele ments\%2Fpbac-guidelines\%2FPBAC4. 3.2.pdf. Accessed July 2011.

42. Churchill D, Morgan J, Torrance G. Quality of life in end-stage renal disease. Periton Dial Bull. 1984;4:20 23.

43. Churchill DN, Torrance GW, Taylor $D W$, et al. Measurement of quality of life in end-stage renal disease: the time trade-off approach. Clin Invest Med. 1987;10:14-20.

44. Hornberger JC, Redelmeier DA, Petersen J. Variability among methods to assess patients' well-being and consequent effect on a cost-effectiveness analysis. J Clin Epidemiol. 1992; 45:505-512.

45. Molzahn AE, Northcott HC, Hayduk L. Quality of life of patients with end stage renal disease: a struc- 
F.S. Mennini et al.

tural equation model. Qual Life Res. 1996;5:426-432.

46. Lenert LA, Hornberger JC. Computer-assisted quality of life assessment for clinical trials. Proceedings of the American Medical Informatics Association, Annual Fall Symposium. 1996:992-996.

47. Sesso R, Yoshihiro MM. Time of diagnosis of chronic renal failure and assessment of quality of life in haemodialysis patients. Nephrol Dial Transplant. 1997;12:2111-2116.

48. Hamel MB, Phillips RS, Davis RB, et al. Outcomes and cost-effectiveness of initiating dialysis and continuing aggressive care in seriously ill hospitalized adults. Ann Intern Med. 1997;127:195-202.

49. Llewellyn-Thomas $H$, Sutherland HJ, Tibshirani R, et al. Describing health states. Methodologic issues in obtaining values for health states. Med Care. 1984;22:543-552.

50. McNeil BJ, Pauker SG, Tversky A. On the framing of medical decisions. In: Bell DE, Raiffa H, Tversky A, eds. Decision Making: Descriptive, Normative, and Prescriptive Interactions. Cambridge, UK: Cambridge University Press; 1988:562-568.

51. Woloshin S, Schwartz LM, Moncur $\mathrm{M}$, et al. Assessing values for health: numeracy matters. Med Decis Making. 2001;21:382-390.

52. Goldstein MK, Miller DE, Davies S, Garber AM. Quality of life assessment software for computer-inexperienced older adults: multimedia utility elicitation for activities of daily living. Proc AMIA Symp. 2002;295-299.

53. Lenert LA, Hornberger JC. Computer-assisted quality of life assessment for clinical trials. Proc AMIA Annu Fall Symp. 1996;992-996. http://www.ncbi.nlm.nih.gov/pmc/ articles/PMC2233008/pdf/procamia afs00002-1025.pdf. Accessed October 2009.

54. Kerlinger FN. Foundations of Behavioral Research. 3rd ed. New York, NY: CBS College Publishing; 1986.
55. Goldstein M, Tsevat J. Applying utility assessment at the "bedside." In: Chapman GB, Sonnenberg FA, eds. Decision
Making in Health Care: Theory, Psychology, and Applications. Cambridge, UK: Cambridge University Press; 2000:313.

Address correspondence to: Dr. Francesco Saverio Mennini, CEIS Sanità (Centre for Health Economics and Management), Faculty of Economics, University Tor Vergata, Via Columbia, 2, 00133 Rome, Italy. E-mail: f.mennini@uniroma2.it 


\section{SUPPLEMENTARY MATERIAL}

This supplementary material was provided by Mennini et al to provide readers with additional information on the article titled "Time trade-off procedure for measuring health utilities loss with human papillomavirusinduced diseases: a multicenter, retrospective, observational pilot study in Italy" (Clin Ther. 2011;33: XXX-XXX).

\section{Overview of the Study}

This pilot study was designed to assess the feasibility of a computer-guided administration of a standardized time trade-off (TTO) procedure to quantify utilities loss in health states affected by HPV-induced pathologies in Italy.

On the basis of a published guideline to design and produce health-state utility instrumentations, ${ }^{1}$ a specific algorithm committed to the computer-guided administration of a questionnaire for the standardized elicitation of utilities in patients with HPV-related high-grade cervical intraepithelial (CIN2-3) lesions was developed. The logic underlying the relationship of the algorithm can be formulated as follows:

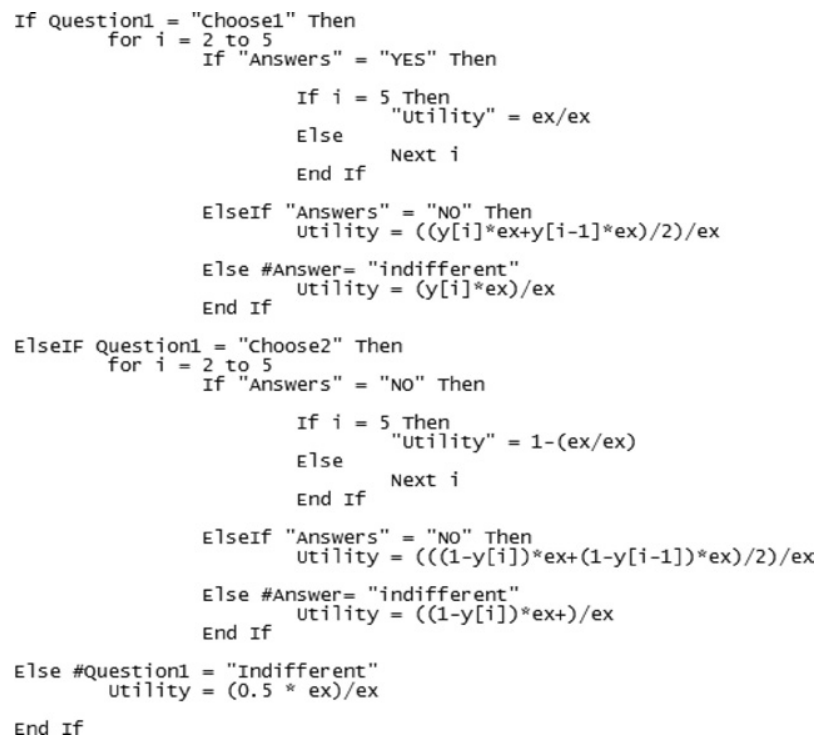

where $e x$ is the life expectancy, $y$ indicates a proportion of the life expectancy, and $i$ indexes the specific question number.

Pathologic conditions of interest were presented using a simply written description and explaining illustrations. The description of disease states were provided as follows.

\section{Cervical Intraepithelial Neoplasia Grades 2 and 3}

Cervical intraepithelial neoplasia (CIN) 2-3 lesions are asymptomatic lesions ("irregularities" or "alterations") of the cervix. They are diagnosed with a Pap test and can be observed with an instrumental examination so-called colposcopy. This condition generally tends to worsen, and a spontaneous regression is very rare. Treatment is based on the removal of the affected tissue with a loop diathermy (ordinary surgical procedure).

After the administration of a local anaesthetic, the gynaecologist removes the portion of cervical tissue involved with a hot wire loop. The procedure is painless, however a sensation of warmth can be produced. The operation does not affect future fertility. This condition can be usually associated with the psychological fear of possible malignant cancer development. In addition, a major concern is given by the risk of possible infection transmitted to the partner and outcomes of future pregnancies.

\section{Cervical Cancer}

Invasive cervical cancer is a lesion commonly bleeding after contact (especially during sexual intercourse) or even spontaneously. When the dimension is relevant, they can cause pain in the lower abdomen and several other symptoms involving the bladder and the rectum. In advanced stages, the bleeding is persistent and difficult to control, a radiating back pain (similar to renal colic) is usual and caused by the tumour mass pushing on close organs.

The treatment is primarily based on surgery, which results in the removal of entire uterus. Depending on the tumour size, the surgical intervention can be performed in two ways.

In the case of very small tumours, the uterus and ovaries are removed (in young women one ovary may be spared whenever possible), no functional disorders occur and the woman can have normal sexual intercourse. With larger tumours, surgery is more radical and involves the removal of lymph nodes and tissues surrounding the cervix, including vessels and nerves of bladder and rectum. The postoperative course is longer with an impairment function of bladder and rectum (difficult urination, urinary retention, difficult defecation). Sexual intercourse is more difficult and or painful. Legs may be swollen. 


\section{Anogenital Warts}

Anogenital warts are lesions caused by a virus (HPV) transmitted mainly through sexual intercourse. The warts can appear in any part of the anogenital area. They appear as small pinkish and fleshy lesions. They are normally asymptomatic, but can sometimes cause itching.

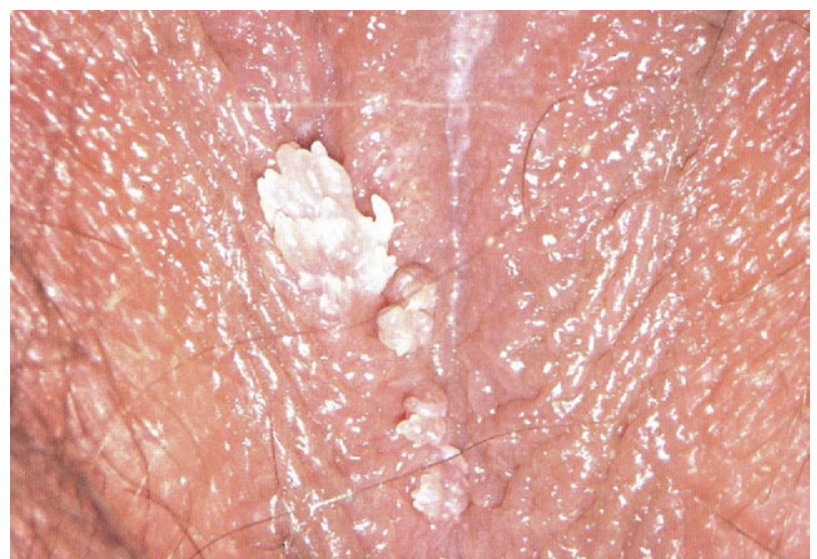

Genital warts located on the skin of the genital area and perineum can be treated by applying some creams or solutions; in some cases, this may cause a burning sensation and redness. If local drugs are not effective, anogenital warts can be surgically removed with a local or a general anaesthesia (if they are very numerous). Warts located on the mucosa of the vulva and vagina

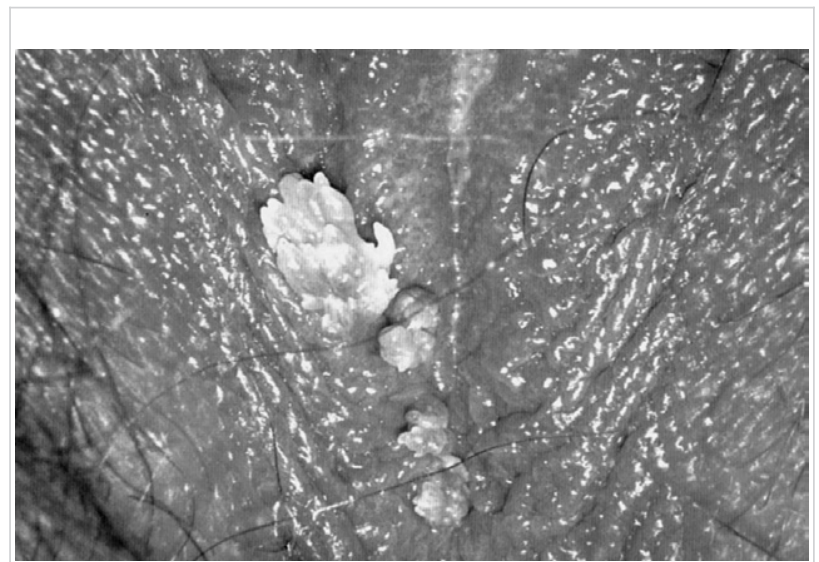

Supplementary Figure 1. Wart lesions localized on external genital area.

cannot be treated with creams or solutions; patients undergo surgical removal. After treatment, the anogenital warts may recur. In these cases, the above-mentioned surgical removal is needed. Anogenital warts are generally associated with anxiety, with a dirty feeling and possible rejection by sexual partner.

\section{REFERENCE}

1. Furlong W, Feeny D, Torrance GW, et al. Guide to design and development of health-state utility instrumentation. http://www.chepa.org/Files/Working Papers/WP 90-9.pdf. Accessed October 2009. 


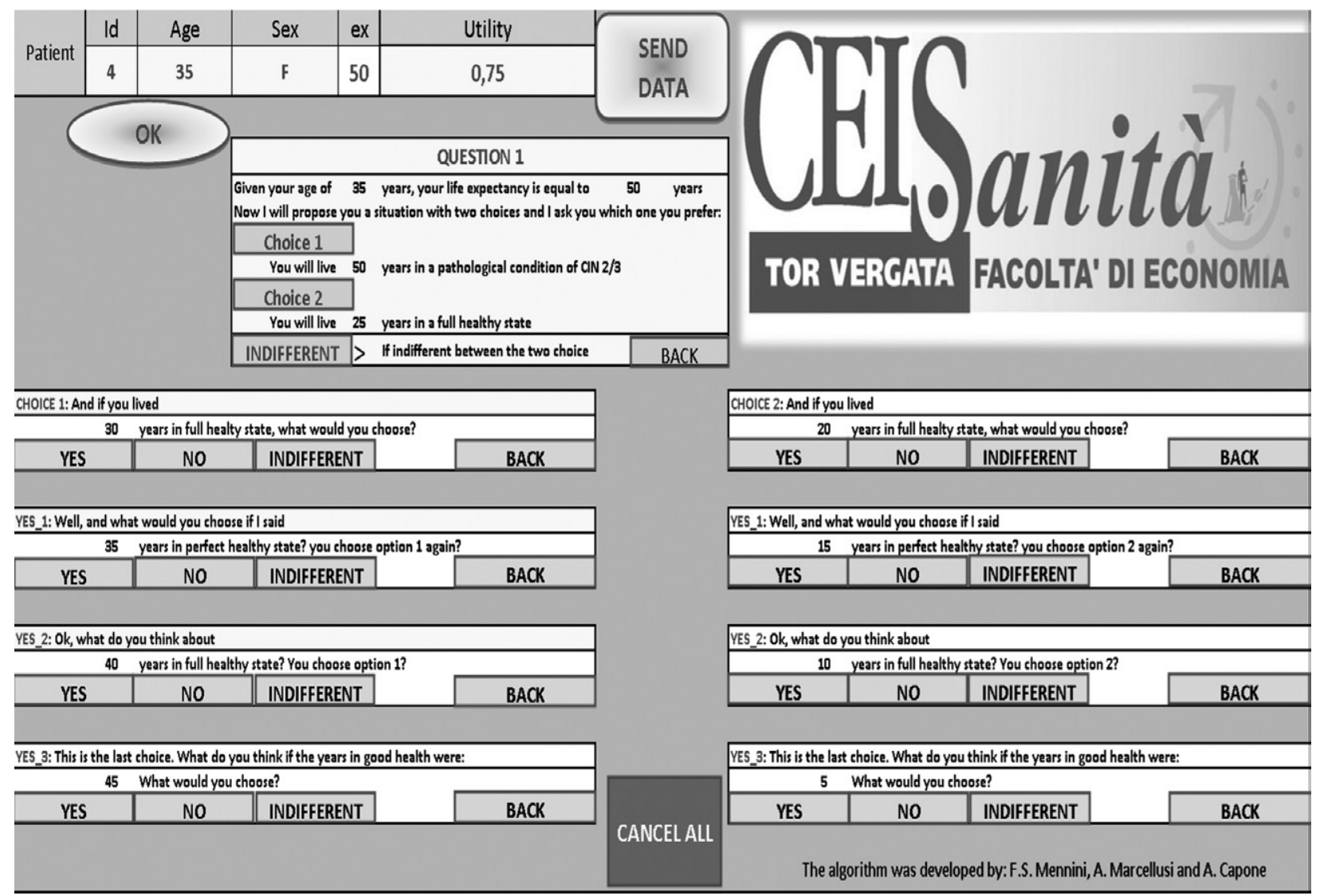

Supplementary Figure 2. Computer-guided administration of a TTO procedure for the standardized elicitation of health state preferences in patients with HPV-related CIN2-3 lesions.
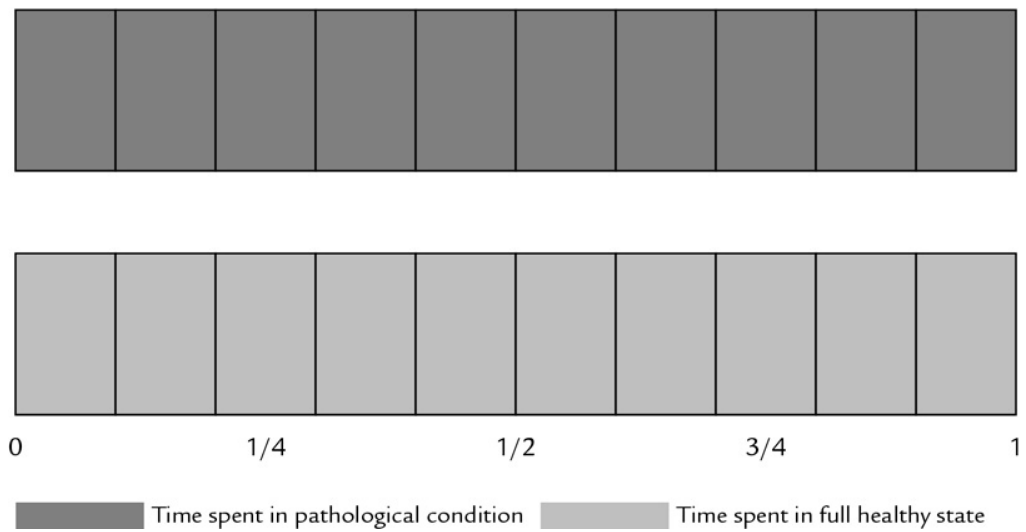

Supplementary Figure 3. Bars visualizing good health state (green, bottom) and bad health state (red, top) to evaluate alternative options. 


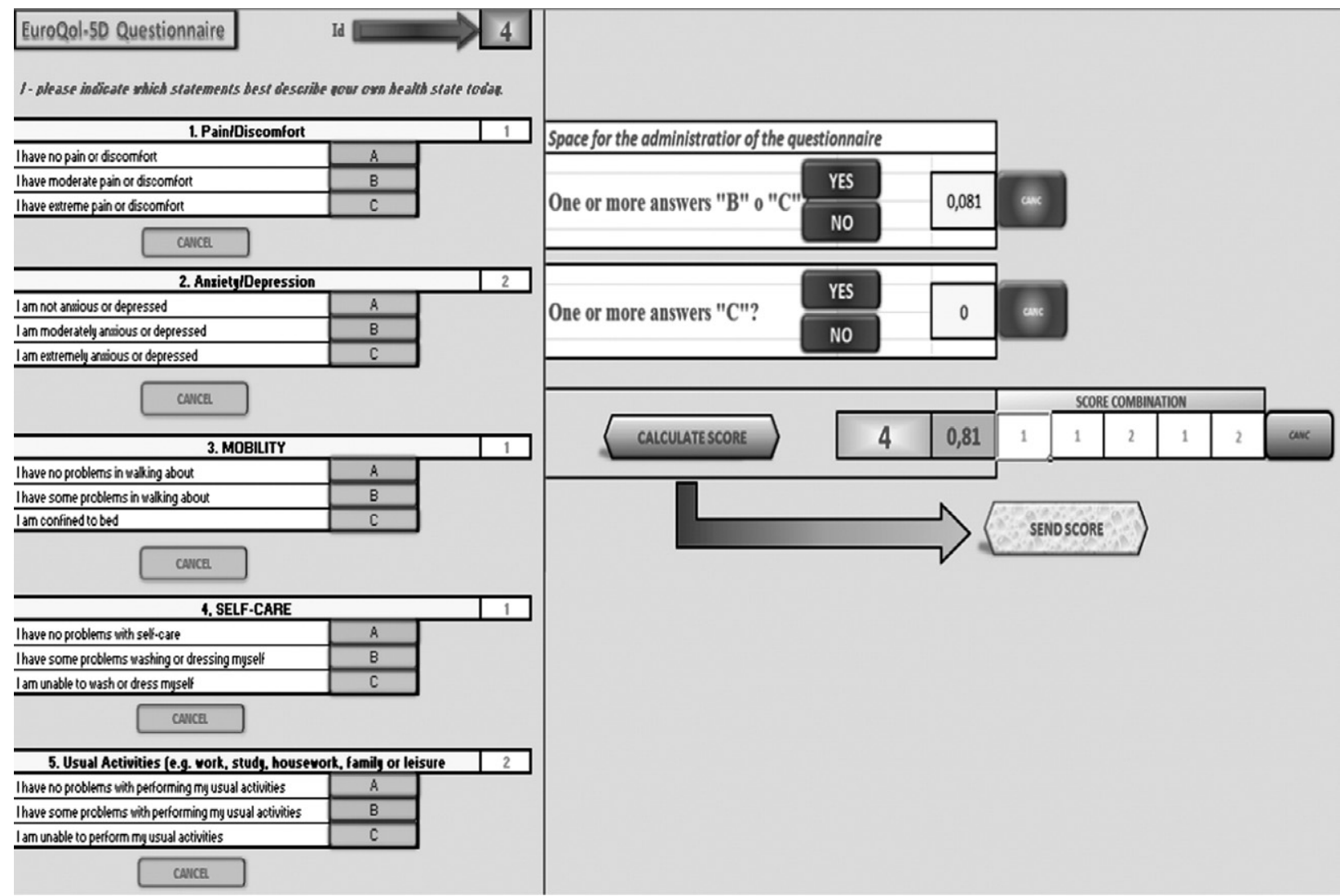

Supplementary Figure 4. Standardized administration system of the EQ-5D. 